\title{
On the defect structures and associated diffraction phenomena in Au nanoparticles
}

Stefan Neumann ${ }^{1}$, Azita Rezvani ${ }^{2}$, Doris Segets ${ }^{3}$ and David Rafaja ${ }^{4}$

${ }^{1}$ Institute of Materials Science, TU Bergakademie Freiberg, Freiberg, Germany, Freiberg, Germany, ${ }^{2}$ Process Technology for Electrochemical Functional Materials, University of Duisburg-Essen, Duisburg, Germany, United States, ${ }^{3}$ Process Technology for Electrochemical Functional Materials and Center for Nanointegration Duisburg-Essen (CENIDE), University of Duisburg-Essen, Duisburg, Germany, United States, ${ }^{4}$ Institute of Materials Science, TU Bergakademie Freiberg, Freiberg, Germany, United States

Au nanoparticles have been studied for decades due to their unique optoelectronic properties that enable high-technology applications, for instance in photovoltaics, sensory probes, drug delivery, electronic conductors and catalysis. Au nanoparticles can be synthesized in a variety of different shapes like decahedron, icosahedron, tetrahedron, triangle, cube, rod, etc. However, the maintenance of most of these shapes requires microstructural defects, such as twins, stacking faults and disclinations. The aim of this work is to investigate the defect structures in differently shaped Au nanoparticles and to correlate these with occurring diffraction phenomena in diffraction patterns of these nanoparticles. Au nanoparticles with a nominal size of about $15 \mathrm{~nm}$ were synthesized by rapidly adding an aqueous solution containing trisodium citrate and tannic acid to an aqueous solution of hydrogen tetrachloroaurate(III) trihydrate after all were heated to $60{ }^{\circ} \mathrm{C}$, followed by boiling of the mixed solution [1]. High-resolution transmission electron microscopy (HRTEM) in combination with fast Fourier transformation (FFT) of the HRTEM micrographs was utilized in order to correlate the shapes of the Au nanoparticles with their atomic structure and in particular to investigate present defect structures and associated diffraction phenomena observed in the FFTs of the HRTEM micrographs. In addition, electron diffraction patterns of faulted structures were simulated by the JEMS software [2]. Careful studies of the FFT/HRTEM patterns of the Au nanoparticles revealed that diffraction spots occur that cannot be indexed as fcc-Au. These "forbidden" diffraction spots are explained on the basis of a conglomeration of microstructural defects, such as stacking faults, twins and disclinations, and their specific orientations with respect to the primary electron beam. For instance, it is demonstrated that diffraction spots in the FFT/HRTEM patterns of Au particles, which were previously attributed to a hexagonal Au phase [3], originate from the interception of the Ewald sphere with reflections elongated along the reciprocal directions $<111>$ as a result of the presence of planar defects on the $\{111\}$ lattice planes of fcc-Au. In conclusion, HRTEM/FFT in combination with the simulation of electron diffraction patterns was successfully applied to explain the occurrence of "forbidden" diffraction spots in the diffraction patterns of Au nanoparticles by specific configurations of microstructural defects and their orientation with respect to the electron beam.

\section{References}

[1] X. Han, J. Goebl, Z. Lu, Y. Yin: Role of Salt in the Spontaneous Assembly of Charged Gold Nanoparticles in Ethanol, Langmuir 27 (2011), 5282-5289.

[2] P. A. Stadelmann: EMS - a software package for electron diffraction analysis and HREM image simulation in materials science, Ultramicroscopy 21 (1987), 131-145.

[3] M. C. Mendoza-Ramirez, H. G. Silva-Pereyra, M. Avalos-Borja: Hexagonal phase into Au plate-like particles: A precession electron diffraction study, Materials Characterization 164 (2020), 110313. 